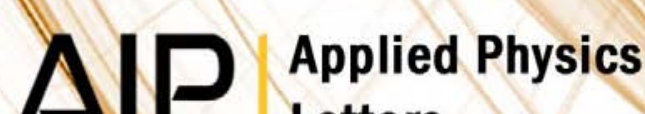 \\ Letters
}

\section{Narrow-line coherently combined tapered laser diodes in a Talbot external cavity with a volume Bragg grating}

David Paboeuf, Gaëlle Lucas-Leclin, Patrick Georges, Nicolas Michel, Michel Krakowski et al.

Citation: Appl. Phys. Lett. 93, 211102 (2008); doi: 10.1063/1.3036896

View online: http://dx.doi.org/10.1063/1.3036896

View Table of Contents: http://apl.aip.org/resource/1/APPLAB/v93/i21

Published by the American Institute of Physics.

\section{Related Articles}

A weakly coupled semiconductor superlattice as a potential for a radio frequency modulated terahertz light emitter

Appl. Phys. Lett. 100, 131104 (2012)

Quantum-dot nano-cavity lasers with Purcell-enhanced stimulated emission

Appl. Phys. Lett. 100, 131107 (2012)

Effect of internal optical loss on the modulation bandwidth of a quantum dot laser

Appl. Phys. Lett. 100, 131106 (2012)

Design of three-well indirect pumping terahertz quantum cascade lasers for high optical gain based on nonequilibrium Green's function analysis

Appl. Phys. Lett. 100, 122110 (2012)

Enhancement of vertical emission in photonic crystal nanolasers

Appl. Phys. Lett. 100, 121117 (2012)

\section{Additional information on Appl. Phys. Lett.}

Journal Homepage: http://apl.aip.org/

Journal Information: http://apl.aip.org/about/about_the_journal

Top downloads: http://apl.aip.org/features/most_downloaded

Information for Authors: http://apl.aip.org/authors

\section{ADVERTISEMENT}

NEW!

iPeerReview

AIP's Newest App

AIP
Authors...

Reviewers...

Check the status of

submitted papers remotely!

AIP Publishing 


\title{
Narrow-line coherently combined tapered laser diodes in a Talbot external cavity with a volume Bragg grating
}

\author{
David Paboeuf, ${ }^{1, a)}$ Gaëlle Lucas-Leclin, ${ }^{1}$ Patrick Georges, ${ }^{1}$ Nicolas Michel, ${ }^{2}$ \\ Michel Krakowski, ${ }^{2}$ Jun Lim, ${ }^{3}$ Slawomir Sujecki, ${ }^{3}$ and Eric Larkins ${ }^{3}$ \\ ${ }^{1}$ Laboratoire Charles Fabry de l'Institut d'Optique, CNRS, Univ Paris-Sud, RD128, \\ 91127 Palaiseau, France \\ ${ }^{2}$ Alcatel-Thales III-V Lab, RD128, 91767 Palaiseau, France \\ ${ }^{3}$ The School of Electronic and Electrical Engineering, University of Nottingham, University Park, \\ Nottingham NG7 2RD, United Kingdom
}

(Received 23 October 2008; accepted 5 November 2008; published online 25 November 2008)

\begin{abstract}
We present the phase locking of an array of index-guided tapered laser diodes. An external cavity based on the self-imaging Talbot effect has been built. A volume Bragg grating is used as the output coupler to stabilize and narrow the spectrum at $976 \mathrm{~nm}$. A power of $1.7 \mathrm{~W}$ is achieved in the in-phase single main lobe mode with a high visibility. We have checked that each emitter is locked to the Bragg wavelength with a $100 \mathrm{pm}$ spectrum linewidth. The experimental results compare well with numerical simulations performed with two-dimensional wide-angle finite difference beam propagation method. (c) 2008 American Institute of Physics. [DOI: 10.1063/1.3036896]
\end{abstract}

High power laser diode arrays are very attractive for applications such as solid-state laser pumping or laser material processing due to a very good overall efficiency and compactness. Inconveniently, the beam quality and the spectrum bandwidth of these devices are often too far from the requirements for demanding applications. A well-known way to enhance the beam quality of a group of many lasers is to induce a phase locking between them. Numerous techniques based on external cavities have been developed in the past with fiber lasers, ${ }^{1} \mathrm{CO}_{2}$ lasers, ${ }^{2}$ and laser diodes. ${ }^{3}$ Among all these techniques, the use of the self-imaging Talbot effect has been established as a very efficient way to produce a highly coherent emission of an array of laser diodes, demonstrating output powers in the watt range in the in-phase mode ${ }^{4-6}$ However, the brightness performance of these lasers is limited by several factors: the free-running operation of the emitters, which limits the operating current range for efficient phase locking, ${ }^{7}$ the use of intracavity filters and magnification optics, which induces extra losses, ${ }^{4}$ and the beam quality of the emitters by themselves. Moreover, the spectrum is often very unstable ${ }^{6}$ and may contribute to the coherency degradation observed at high current. Recently, volume Bragg gratings (VBGs) have appeared to become essential optical components and have been extensively used for wavelength stabilization and spectrum narrowing in external cavities. ${ }^{8,9}$ Phase locking of two laser diodes by use of two VBGs has also been demonstrated but with the drawback of a nonscalable configuration. ${ }^{10}$ In this work, we present the development of an external cavity based on the Talbot self-imaging effect to phase-lock an array of ten tapered laser diodes. Furthermore, a VBG is used as the output coupler to stabilize and narrow the spectrum.

In our experiments, the laser source is an array of ten index-guided tapered lasers emitting around $975 \mathrm{~nm}$ with a pitch $p=100 \mu \mathrm{m}$, which results in a Talbot distance $\left(2 p^{2} / \lambda\right)$ of $20.4 \mathrm{~mm}$. The active layer consists of a strained GaInAs quantum well embedded in a large optical cavity. The lateral

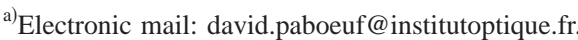

structure of the emitters is a tapered ridge with a narrow angle $\left(<1^{\circ}\right)$ and an overall cavity length of $2.5 \mathrm{~mm}$. The tapered design allows high power emission together with a single transverse mode operation; thanks to the widened active section. Such an array has already demonstrated $4 \mathrm{~W}$ at $6 \mathrm{~A},{ }^{11}$ with a far-field full width at $1 / e^{2}$ in the slow axis of $2.7^{\circ}$ and a $M^{2}$ parameter $<2$. The rear facet is high-reflection coated $(R>90 \%)$. Thanks to the antireflection coating $(R$ $<10^{-3}$ ) on the front facet of the laser array, no laser emission but only amplified spontaneous emission is observed without external cavity. This feature is very important to avoid competition between the free-running operation of the emitters and the coherent emission induced by the external cavity.

Our experimental setup is shown in Fig. 1. The beam from the laser array is collimated in the fast-axis direction with a high numerical aperture acylindric lens (Doric Lenses D142-375). The remaining divergence is lower than $3 \mathrm{mrad}$. During the alignment, the lens is fixed to a three-axis $(X Y Z)$ translation stage; then it is definitely glued to its optimal position with an epoxy adhesive (Araldite) and the translation stage is removed. A VBG from ONDAX, Inc. is used as the external mirror. It is designed to reflect $40 \%$ at $976 \mathrm{~nm}$ with a spectral bandwidth $\Delta \lambda=0.3 \mathrm{~nm}$. The angular acceptance of the VBG is $\Delta \theta=3^{\circ}$ (full width at $1 / e^{2}$ ), which is

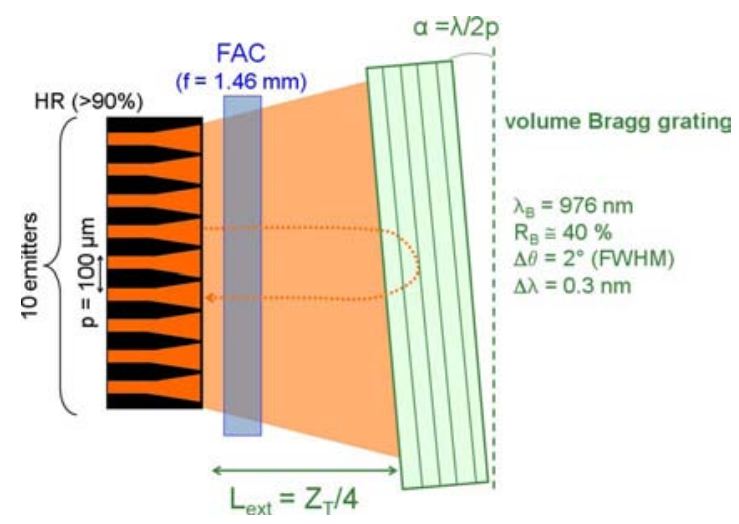

FIG. 1. (Color online) External cavity setup. 


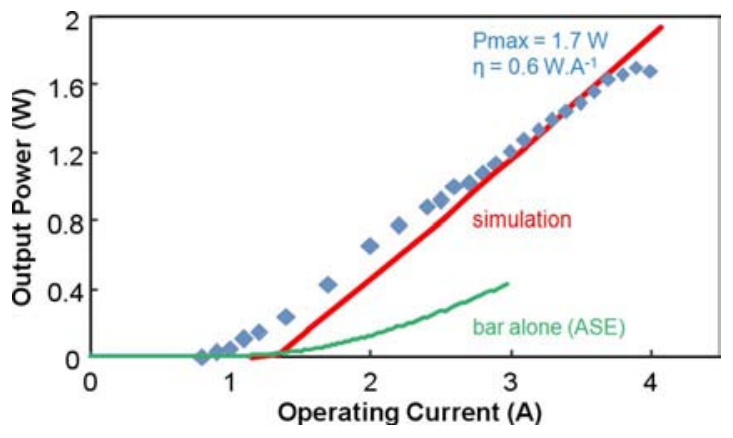

FIG. 2. (Color online) Output power characteristic of the laser in external Talbot cavity (blue diamond: experiment; red line: numerical simulation) and of the bar alone (green line).

larger than the divergence of the laser array in both directions after fast-axis collimation. The diffraction efficiency of the VBG will then remain close to maximum. ${ }^{9}$ The VBG is located at a quarter of the Talbot distance from the front facet for maximum discrimination between the in-phase and the out-of-phase modes. This setup results in a very compact ( $5 \mathrm{~mm}$ long) and simple cavity due to the choice of the array pitch, with only one intracavity element for limited losses. The whole setup is mounted on a copper block and actively thermally regulated.

With the VBG as the output coupler of the cavity, a threshold of $0.9 \mathrm{~A}$ and a power of $1.7 \mathrm{~W}$ at an operating current of 3.9 A have been obtained. The slope efficiency is $0.6 \mathrm{~W} \mathrm{~A}^{-1}$ (see Fig. 2). With untilted VBG, the cavity operates in the out-of-phase mode, which exhibits two main peaks in the far field. By tilting the VBG by an angle $\alpha$ $=\lambda / 2 p$, the in-phase mode is selected. The slow-axis angular distribution of the laser was measured with a chargecoupled dence camera at the Fourier plane of a cylindrical collimating lens. The far-field profile exhibits interference peaks with a maximum at $\theta=0^{\circ}$, which is a clear evidence of the phase locking in the in-phase mode (see Fig. 3). The full width at half maximum of each peak is $1.2 \mathrm{mrad}$, which is very close to the diffraction angular limit of the array $(1 \mathrm{mrad})$. At high output powers $(P>1.2 \mathrm{~W})$, the incoherent contribution of the free-running array operation induces a parasitic pedestal in the far-field distribution; moreover the interference peaks are slightly broadened to $2.5 \mathrm{mrad}$.

The maximum output power is only limited by the thermal rollover of the array, which appears at $4 \mathrm{~A}$ under our

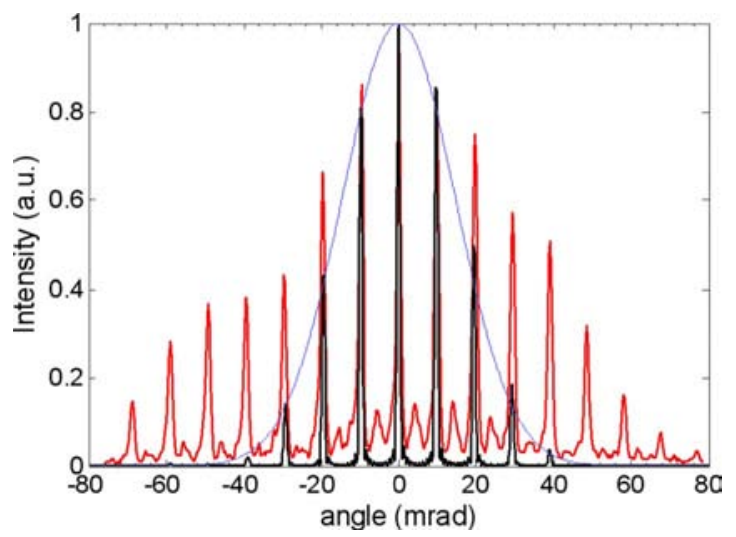

FIG. 3. (Color online) Far-field profiles; red line-experimental measurement, black line-numerical result, and blue line-theoretical far field of a Gaussian emitter $(I=3 \mathrm{~A}, P=1.2 \mathrm{~W})$. experimental conditions. This maximum output power value roughly corresponds to $60 \%$ of the power obtained in freerunning operation $(2.8 \mathrm{~W})$ at the same current with a similar chip, ${ }^{11}$ which means that the external cavity configuration extracts the maximum available power from the laser bar. The envelope of the far field presents some distortion at high power, which is attributed to a degradation of the beam quality of the emitters. We have checked that similar performance is obtained with a plane mirror $(R=40 \%)$ as the output coupler instead of the VBG, which shows the efficiency of the VBG to provide a coupling between the emitters together with a strong spectral control. In order to characterize the coherence quality of our cavity, we have calculated the visibility defined as follows:

$$
V=\frac{I_{\max }-I_{\min }}{I_{\max }+I_{\min }} .
$$

A visibility as high as $80 \%$ has been measured at low power (800 mW, I=2.5 A), which demonstrates the high degree of coherence induced by the Talbot external cavity. At higher currents, the visibility decreases to $70 \%$ due to increased incoherent free-running contribution.

Simulations of the behavior of the cavity have been carried out with an iterative approach based on the Fox-Li method. The optical beam is propagated back and forth inside the cavity until a steady state power and an amplitude profile are reached. The model of the tapered laser bar combines the electrical and optical models in the same way as described by Lim et al. ${ }^{12}$. The isothermal electrical model consists of the one-dimensional unipolar carrier-diffusion equation, where the injection current density $J(x)$ is assumed constant inside the stripe. This equation is solved for the carrier density distribution so that spatial hole burning and carrier lensing effects are included. The optical fields in the tapered laser are modeled with the two-dimensional wideangle finite difference beam propagation method using the effective index approximation. The free-space propagation, including reflection from a tilted plane output mirror $(R$ $=40 \%$ ), in the external cavity, is modeled using the Fresnel diffraction equations. The optical beam is assumed to be TE polarized and monochromatic. In Fig. 2, we compare the experimental $L-I$ characteristic to the simulated one. For each current value we checked that the program converges to the in-phase supermode. Although the simulation results exhibit a higher threshold and slope efficiency, the two characteristics are in good agreement. The differences are attributed to disparities between the theoretical laser parameters used in the model and their effective values in the experiments. In addition, the use of a unipolar, isothermal model, which does not properly account for current spreading and self-heating effects, could also contribute to the discrepancy. The far-field distribution obtained at $3 \mathrm{~A}$ is compared to the experimental one in Fig. 3. These results show that the interference peaks have the same width of $1.2 \mathrm{mrad}$ in both cases. The theoretical envelope of the far field is close to a Gaussian profile with a full width at $1 / e^{2}$ of $40 \mathrm{mrad}$ related to the dimension of the emitters. It differs from the experimental one, which is larger, because of the presence of a multimode emission from the tapered diodes.

In order to characterize the spectrum of the laser array, we have separately measured the spectrum of each emitter with an optical spectrum analyzer (resolution of $70 \mathrm{pm}$ ). The 


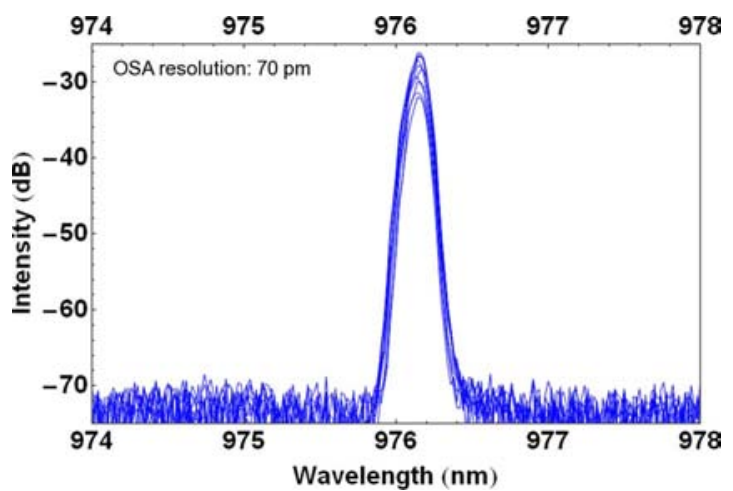

FIG. 4. (Color online) Superimposed spectra of the ten emitters measured with a $70 \mathrm{pm}$ resolution optical spectrum analyzer $(I=2.8 \mathrm{~A}, P=1 \mathrm{~W})$.

results for $I=2.8 \mathrm{~A}$ are shown in Fig. 4. Obviously, each emitter is locked at the Bragg wavelength, with no evidence of parasitic lines and no relative emitter drift. A highresolution $(10 \mathrm{pm})$ spectral measurement of a few emitters shows that the spectral linewidth is actually below $0.1 \mathrm{~nm}$ irrespective of the operating current $(I<4 \mathrm{~A})$.

In summary, we have demonstrated an original concept of external cavity exploiting the properties of VBGs. A very simple and compact external cavity based on the Talbot selfimaging effect has been developed to perform the phaselocking of an array of tapered laser diodes. The careful design of the cavity parameters allows a highly coherent operation on a large power range. A maximum output power of $1.7 \mathrm{~W}$ is obtained in the in-phase array supermode. The wavelength of every emitter in the array is locked to the Bragg wavelength regardless of the operating conditions. A whole spectral bandwidth of $0.1 \mathrm{~nm}$ has been measured. Increase in power should be possible with improved laser di- odes and a lower reflectivity grating. This concept could be easily adapted to other kinds of semiconductor laser arrays, such as gain-guided tapered laser diodes and Slab-Coupled Optical Waveguide Lasers. ${ }^{6}$

This work was supported within the European project BRIGHTER.EU under Grant No. FP6-IST-035266. D. Paboeuf thanks the French Ministry of Defense (Délégation Générale de l'Armement) for funding his Ph.D. N. M. and M. Kr. thank M. Calligaro, M. Lecomte, O. Parillaud, Y. Robert, M. Carbonnelle, and C. Dernazaretian for the realization of tapered lasers.

${ }^{1}$ C. J. Corcoran and F. Durville, Appl. Phys. Lett. 86, 201118 (2005).

${ }^{2}$ A. F. Glova, Quantum Electron. 33, 283 (2003).

${ }^{3}$ C. J. Chang Hasnain, J. Berger, D. R. Scifres, W. Streifer, J. R. Whinnery, and A. Dienes, Appl. Phys. Lett. 50, 1465 (1987)

${ }^{4}$ R. Waarts, D. Mehuys, D. Nam, D. Welch, W. Streifer, and D. Scifres, Appl. Phys. Lett. 58, 2586 (1991).

${ }^{5}$ J. R. Leger and G. Mowry, Appl. Phys. Lett. 63, 2884 (1993).

${ }^{6}$ R. K. Huang, B. Chann, L. J. Missagia, S. J. Augst, R. B. Swint, J. P. Donnelly, A. Sanchez-Rubio, and G. W. Turner, presented at the Conference on Lasers and Electrooptics (2008).

${ }^{7}$ I. Hassiaoui, N. Michel, G. Bourdet, R. McBride, M. Lecomte, O. Parillaud, M. Calligaro, M. Krakowski, and J. P. Huignard, Appl. Opt. 47, 746 (2008).

${ }^{8}$ G. B. Venus, A. Sevian, V. I. Smirnov, and L. B. Glebov, High-Power Diode Laser Technology and Applications III (SPIE, Bellingham, WA, 2005), Vol. 5711, pp. 166-176.

${ }^{9}$ G. Lucas-Leclin, D. Paboeuf, P. Georges, J. Holm, P. Andersen, B. Sumpf, and G. Erbert, Appl. Phys. B: Lasers Opt. 91, 493 (2008).

${ }^{10}$ G. B. Venus, A. Sevian, V. I. Smirnov, and L. B. Glebov, Opt. Lett. 31, 1453 (2006)

${ }^{11}$ G. L. Bourdet, I. Hassiaoui, R. McBride, J. F. Monjardin, H. Baker, N. Michel, and M. Krakowski, Appl. Opt. 46, 6297 (2007).

${ }^{12}$ J. J. Lim, T. M. Benson, and E. C. Larkins, IEEE J. Quantum Electron. 41, 506 (2005). 